
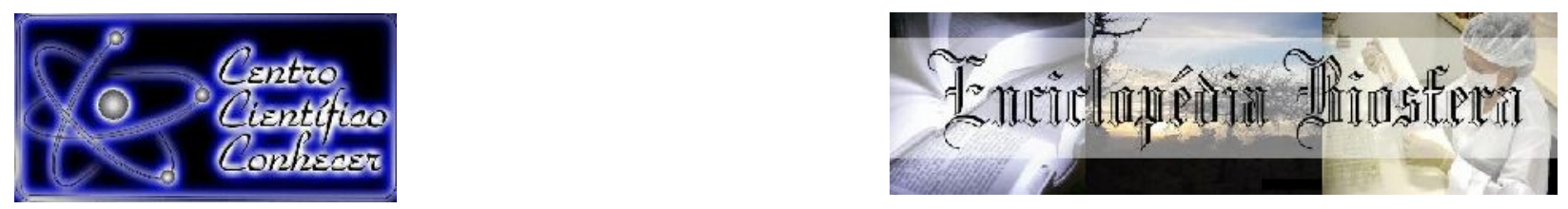

\title{
APLICAÇÃO DOS CONCEITOS SDN, DEVOPS E INFRAESTRUTURA COMO CÓDIGO NO PROCESSO DE ENSINO E APRENDIZAGEM DE CIÊNCIAS E ENGENHARIAS
}

Anderson de Castro Lima', Sandro César Silveira Jucá ${ }^{2}$, Pedro Bruno Silva Lemos³, Solonildo Almeida da Silva ${ }^{4}$

1 Doutorando em Ensino (RENOEN) pelo Instituto Federal de Educação, Ciência e Tecnologia do Ceará (IFCE), Fortaleza-CE, Brasil. (anderson@ifce.edu.br)

2 Doutor em Engenharia Elétrica pela Universidade Federal do Ceará, Fortaleza-CE, Brasil.

${ }^{3}$ Doutorando em Ensino (RENOEN) pelo Instituto Federal de Educação, Ciência e Tecnologia do Ceará (IFCE), Fortaleza-CE, Brasil.

${ }^{4}$ Doutor em Educação, Professor do Instituto Federal de Educação, Ciência e Tecnologia do Ceará (IFCE), Maracanaú-CE, Brasil.

\section{Recebido em: 15/08/2021 - Aprovado em: 15/09/2021 - Publicado em: 30/09/2021 DOI: 10.18677/EnciBio_2021C4}

trabalho licenciado sob licença Creative Commons Attribution-NonCommercial-NoDerivatives 4.0 International License.

\section{RESUMO}

A Internet é uma ferramenta de trabalho e estudo fundamental para os dias atuais, esta proporciona acesso a um vasto conhecimento e a comunicação em tempo real dentre outras possibilidades, porém com ela surge a necessidade de profissionais que possam mantê-la em funcionamento constante exigindo que o tempo de falha seja o menor possível, desse modo as ações tradicionais de configuração e manutenção de equipamento por equipamento está sendo substituída por códigos de programação que com poucos comandos fazem o trabalho em minutos que antes poderia durar horas ou dias. Em conformidade com essa discussão, este trabalho trata-se de uma revisão bibliográfica a respeito de três abordagens, a saber: redes definidas por software do inglês Software Defined Network - SDN, a cultura DevOps e o conceito de infraestrutura como código do inglês infrastructure as a code - laaC que em conjunto proporcionarão aos alunos de ciências e engenharias uma base sólida de conhecimento capaz de suprir essa nova demanda. Esses elementos possuem uma carga teórica e prática muito grande e para melhor assimilação dos estudantes eles serão trabalhados usando a metodologia de ensino baseada em problemas - ABP em conjunto com o framework de gestão ágil SCRUM para maximizar tanto o conhecimento técnico como o comportamento interpessoal exigidas no mercado atual.

PALAVRAS-CHAVE: DevOps, laaC, SDN. 


\title{
APPLICATION OF SDN, DEVOPS AND INFRASTRUCTURE AS A CODE IN SCIENCE AND ENGINEERING TEACHING AND LEARNING PROCESS
}

\begin{abstract}
The Internet is a fundamental work and study tool for today, it provides access to vast knowledge and real-time communication, among other possibilities, but with it comes the need for professionals who can keep it in constant operation, demanding that the failure time is as short as possible, in this way the traditional actions of configuration and maintenance of equipment by equipment are being replaced by programming codes that with few commands do the work in minutes that previously could take hours or days. In accordance with this discussion, this work is a literature review regarding three approaches, namely: Software Defined Network - SDN, the DevOps culture and the concept of infrastructure as code - laaC, which together will provide science and engineering students with a solid knowledge base capable of meeting this new demand. These elements have a very large theoretical and practical load and for better assimilation of students they will be worked using the problem-based çearning methodology - PBL in conjunction with the SCRUM agile management framework to maximize both the technical knowledge and the interpersonal behavior required in the current market.
\end{abstract}

KEYWORDS: SDN, DevOps, laaC.

\section{INTRODUÇÃO}

A Internet faz parte do cotidiano das pessoas há muito tempo. Empresas, instituições, todos usam a rede mundial para inúmeras tarefas, porém muitos esquecem que, para a Internet funcionar minimamente, ou seja, entregando a informação solicitada por um usuário, precisa de uma infraestrutura de rede presente, seja cabeada ou sem fio, sendo composta por computadores, roteadores, modens, switches, pontos de acesso e muitos outros. O problema é quando um desses equipamentos falham ou é mal configurado. Com o atual estilo de vida, pode ocasionar segundos de espera acarretando prejuízo para algumas pessoas ou empresas (SHAH; DUBARIA, 2019).

A infraestrutura da rede é vital para a existência da Internet e esta precisa se aprimorar continuamente com o surgimento constante de novos serviços e da pressão de funcionamento ininterrupto. Como exemplo para esse novo tipo de demanda, pode-se citar as casas inteligentes onde, antes mesmo do usuário chegar à residência, o ar-condicionado poderá ser ligado para que a temperatura interna esteja adequada para o conforto ao entrar. No mundo corporativo, redes programáveis é uma demanda crescente, principalmente com o advento da computação em nuvem, onde um serviço pode exigir um tipo de configuração e mudar de uma hora para outra, exigindo que esta se adapte na mesma velocidade (RAY; KUMAR, 2021).

A computação em nuvem só é possível através da programação e virtualização dos equipamentos, mas isso reflete também na rede, assim estas também devem ser programáveis, trazendo à tona os conceitos de Redes Definidas por Software, Infraestrutura como Código e DevOps que passarão a ser aplicados no ensino das engenharias a fim de preparar os alunos para demandas do mercado (SHAH; DUBARIA, 2019). Adicionalmente, esse projeto contemplará a utilização do 
conceito de redes sensíveis ao contexto em que regras podem criar novas regras baseadas nas modificações percebidas no ambiente.

Com o advento da Internet das coisas, do inglês Internet of things - IoT, a tendência é que haja um aumento expressivo do tráfego na rede e devido a heterogeneidade dos equipamentos que formarão essa estrutura, as redes definidas por software, do inglês Software Defined Networks - SDN se apresentam como soluções interessante tanto para viabilizar a comunicação entre os equipamentos como para gerenciar o tráfego dessas redes de forma a minimizar o grande volume de dados trafegados (CARAGUAY et al., 2014). Um dos mecanismos propostos para serem implementados no ensino aplicará o controle de acesso em Redes Definidas por Software sensíveis ao contexto para ambientes cabeadas e sem fio em conjunto com dispositivos loT. Para um melhor entendimento, a sigla SDN será usada para se referenciar a Redes Definidas por Software em todo o resto do trabalho.

A aprendizagem baseada em problema (ABP) em conjunto com a gestão de projetos ágeis com foco no framework SCRUM serão os métodos de ensinos aplicados para que a teoria se transforme em algo prático e nesse sentido, nos últimos anos o IFCE Maracanaú, instituição onde será aplicado o projeto, trabalhou para que através do empreendedorismo, as pesquisas fossem transformadas em benefício para a sociedade através da criação de startups, como demonstrado no artigo do livro de Bauerle et al. (2020).

Dessa forma, o objetivo geral do estudo é analisar, mediante realização de fundamentação teórica, o uso de conceitos como SDN, DevOps e infraestrutura como código no ensino, propondo o uso da ABP com o SCRUM na criação de uma estrutura de rede programável.

Em termos metodológicos, o presente trabalho, destarte, tratou-se de uma pesquisa do tipo revisão bibliográfica que objetivou discutir as estratégias de ensino que relacionem o controle de acesso em Redes Definidas por Software sensíveis ao contexto para ambientes cabeadas e sem fio.

A revisão bibliográfica é caracterizada pela análise de produções científicas e acadêmicas que passaram por tratamento analítico (GIL, 2008). Sendo assim, nesta pesquisa analisou-se, prioritariamente, artigos científicos e livros que abordam propostas de ensino de $S D N$ centradas na utilização da metodologia de Aprendizagem baseada em problemas do inglês Problem Based Learning - PBL, e no SCRUM, um conjunto de ferramentas voltadas para o desenvolvimento ágil de projetos.

Ademais, convém mencionar que a análise bibliográfica foi pautada em obras publicadas nos últimos cinco anos (2016-2021) e a seleção de referências foi realizada nas bases de dados acadêmicas e científicas Scientific Electronic Library Online (Scielo) e Google Scholar. Por fim, destaca-se que o processo de identificação e seleção das referências foi realizado no decorrer do mês de julho de 2021.

\section{REFERENCIAL TEÓRICO}

O referencial teórico descrito neste trabalho objetiva discutir propostas didáticas que proporcionem a inserção de códigos de programação no ensino de Ciências e Engenharias. Em adicional, é importante ressaltar que foram selecionadas estratégias de ensino que fomentem a atuação autônoma e crítica dos discentes no processo de aprendizagem. Desse modo, as seções apresentam 
discussões relacionadas às temáticas: Software Defined Network - SDN, Cultura DevOps, Infraestrutura como código, Controle de acesso, Aprendizagem Baseada em Problemas - ABP e SCRUM.

\section{Software Defined Network - SDN}

A ideia de redes programáveis não é recente, com os computadores no início dos anos 1980, ocorreu uma separação entre Hardware e Software, ou seja, antes comprava-se um computador que já vinha com o sistema operacional, após essa separação surgiu a Microsoft e bem depois as distribuições Linux, porém, com os ativos de rede isso ainda não ocorreu. Na compra de um roteador, switch ou ponto de acesso, o sistema operacional já vem instalado permitindo apenas que o usuário possa efetuar configurações limitadas as opções que o fabricante disponibiliza. Com o $S D N$, fazendo uma explicação simplista, será semelhante ao ocorrido com os computadores, os equipamentos de rede passarão a não terem mais o software de decisão embarcado, isso ficará em um computador externo, chamado de controlador com o sistema operacional que poderá ser escolhido pelo usuário, facilitando o gerenciamento e a inovação segundo Chica et al. (2020).

O SDN consiste em três entidades: o controlador, o switch compatível com SDN e o canal de comunicação segura entre o controlador e o switch. Desse modo, o equipamento de rede passará a ter um papel apenas de encaminhamento de pacotes, ficando no chamado plano de dados, que consiste em possuir tabelas de encaminhamentos contendo as orientações para envio dos pacotes, as ações a serem tomadas para um determinado fluxo e um canal de comunicação segura que será usado para receber configurações do controlador ou encaminhar para este pacotes que não possuam informações na tabela de encaminhamento do switch, a tomada de decisão fica a cargo desse controlador que estará no plano de controle (CHICA et al., 2020).

A comunicação entre o controlador e 0 ativo de rede poderá utilizar o protocolo OpenFlow (MCKEOWN et al., 2008) um dos mais adotados para redes $S D N$, que tem como função acessar a tabela de encaminhamento do equipamento de rede e inserir as informações enviadas pelo controlador. A característica principal do protocolo OpenFlow é a separação clara entre os planos de dados e controle nos elementos de encaminhamento de dados. O plano de dados cuida do encaminhamento de pacotes com base em regras simples (chamadas de ações) associadas a cada entrada da tabela de encaminhamento do comutador de pacotes (um switch ou roteador). Essas regras definidas pelo OpenFlow incluem: (i) encaminhar o pacote para uma porta específica do dispositivo, (ii) alterar parte de seus cabeçalhos, (iii) descartá-lo, ou (iv) encaminhá-lo para análise por um controlador de rede. Já o plano de controle permite ao controlador da rede programar as entradas desta tabela de encaminhamento com padrões que identifiquem fluxos de interesse e as regras associadas a eles (BENZEKKI et al., 2016).

O Controlador tem sido comparado a um sistema operacional de rede o qual fornece uma interface de programação para a gestão da rede. Enquanto isso simplifica as tarefas de execução e gestão de políticas. A comunicação deve ser cuidadosamente protegida entre o controle e os elementos de encaminhamento de rede. Devido o $S D N$ ter chamado a atenção do mercado, surgiu uma variada gama de controladores, sendo o NOX (GUDE et al., 2008) um dos primeiros, desenvolvido 
e programado em $\mathrm{C}++_{+}$, posteriormente baseado no NOX foi criado o POX (POX, 2021) implementado em python. Existem outros tipos de controladores, mas ultimamente um tem chamado a atenção por estar recebendo o apoio de grandes fabricantes como CISCO, HP e IBM, seu nome é OpenDaylight (OPENDAYLIGHT, 2021) um controlador desenvolvido e programável em Java.

A ideia de uma rede inteligente com gerenciamento centralizado em um Controlador $S D N$, faz com que esta possua uma visão global, favorecendo não apenas o gerenciamento em redes cabeadas, mas poderá ser de grande ajuda em Redes Mesh sem fio, na qual os equipamentos são interconectados através de ondas eletromagnéticas e para Redes de sensores sem fio (BRITO et al., 2014).

As redes que usam o $S D N$ são programáveis de forma homogênea entre os dispositivos que compõem a rede, já na tradicional a forma de configurar varia de fabricante para fabricante e pode até ser diferente dependendo do modelo de mesmos fabricantes. Como SDN a gerência fica centralizada, melhorando assim o acompanhamento do comportamento da rede e facilitando a tomada de decisão para a correção de algum problema de forma mais rápida do que em redes tradicionais. Devido a rede $S D N$ ser centralizada e programável, esta reduz os riscos de erros de configuração pois, os comandos são acionados no ponto de controle central, diferente das redes clássicas que as configurações são feitas em cada equipamento de forma independente, com isso os autores mostram o quão benéfico é a adoção de redes SDN em comparação com as redes tradicionais (BENZEKKI et al., 2016).

Em resumo no artigo de Benzekki et al., (2016) os autores fazem uma comparação entre as redes convencionais e as redes que utilizam o paradigma SDN que foi adaptado para o quadro 1 :

QUADRO 1: Comparação entre redes SDN e Clássicas

\begin{tabular}{lcc}
\hline Características & $\begin{array}{c}\text { Redes } \\
\text { SDN }\end{array}$ & $\begin{array}{c}\text { Redes } \\
\text { Clássicas }\end{array}$ \\
\hline Serem programáveis & $\mathrm{X}$ & \\
\hline Controle centralizado & $\mathrm{X}$ & \\
\hline Exposta a erros de configuração & & $\mathrm{X}$ \\
\hline Facilidade de adaptação a mudanças & $\mathrm{X}$ & \\
\hline Melhoria da performance & $\mathrm{X}$ & \\
\hline Configurações mais eficientes & $\mathrm{X}$ & \\
\hline Aprimoramento no gerenciamento & $\mathrm{X}$ & \\
\hline
\end{tabular}

Fonte: Benzekki et al. (2016)

\section{Cultura DevOps}

A maioria das redes de computadores em empresas privadas ou públicas e em instituições de ensino são gerenciadas equipamento por equipamento via linha de comando ou interface web de configuração, ambos disponibilizados da forma que o fabricante do equipamento deseja, ou seja, não existe padrão para configuração entre dispositivos de marcas diferentes. Nesse tipo de rede, pequenas mudanças costumam ter grandes impactos e podem provocar comportamentos indesejados (SHAH; DUBARIA, 2019).

As infraestruturas de redes atuais podem ser consideradas como estáticas, demandam atividades mais complexas para se ajustar a qualquer tipo de mudança, seja solicitada ou não pelos usuários. Para minimizar a implementação dessas 
mudanças, são utilizadas soluções de redes programáveis como o paradigma de Redes Definidas por Software ou SDN, fazendo com que a operação se torne muito mais automatizada e simplificada. Com esse tipo de rede, novos recursos podem ser implementados pressionando um único botão. Com essa nova tecnologia, sistemas automatizados podem realizar alterações de configuração, realizando testes em cada estágio para garantir que as essas mudanças de rede não resultem em tempo de inatividade e, finalmente, implantem essas configurações no dispositivo desejado (SHAH; DUBARIA, 2019).

Com base no que foi discutido anteriormente, pode-se afirmar que as alterações na rede estão diretamente relacionadas com o aumento da frequência de interrupções, considerando que cada interrupção pode levar muitas horas para ser resolvida e que a maioria dos profissionais de rede ainda estão verificando manualmente a infraestrutura da rede depois que os problemas são percebidos. Isso, portanto, leva a concluir que é preciso encontrar uma forma melhor de verificar mudanças na rede indo de encontro com o conceito de deixá-las programáveis e automatizadas. Para esta nova era serão necessários profissionais que possam estar aptos a trabalhar baseado na cultura DevOps (SHAH; DUBARIA, 2019).

Primeiramente, DevOps não é uma nova função que apareceu nas equipes de tecnologia da informação, ela é composta por duas siglas: DEV e OPS. O DEV representa a equipe de desenvolvimento, responsável por desenvolver software baseado em demandas provenientes dos usuários e a sigla OPS representa a equipe de operação, responsável por receber esse programa construído pela equipe de desenvolvimento e colocar em operação para que os usuários que a demandaram possam utilizar (DYCK et al., 2015).

Existem duas equipes que precisam trabalhar em conjunto a fim de disponibilizar um determinado serviço, advindo de um software, para usufruto dos usuários, porém, com o tempo sendo cada vez mais valioso, o período gasto entre a desenvolvimento do software e a entrada em operação em alguns casos deve ser quase zero, principalmente quando é uma atualização em um software que já está em operação, assim algumas empresas acabam tendo prejuízo na demora desse processo (BASHER, 2019).

O DevOps, é uma cultura que visa estabelecer uma mentalidade que se concentra em permitir a colaboração mais próxima entre a equipe de desenvolvimento (Dev) com a equipe operacional (Ops), definindo o objetivo comum de desenvolver softwares de alta qualidade e operar em sistemas resilientes. (cita artigo) Vale salientar que, embora DevOps seja uma abreviatura de desenvolvimento e operações, ele não se limita a essas duas equipes, pois o objetivo é que existam profissionais capazes de exercer as duas funções ao mesmo tempo e que ainda possam ter uma visão do negócio, ou seja, do impacto, financeiro ou não, que essa solução terá na empresa ou instituição (LEITE et al., 2019). A fim de facilitar o entendimento, a figura 1 apresenta o ciclo do DevOps, note que o símbolo do infinito indica que esse ciclo é interminável. 
FIGURA 1: Ciclo DevOps

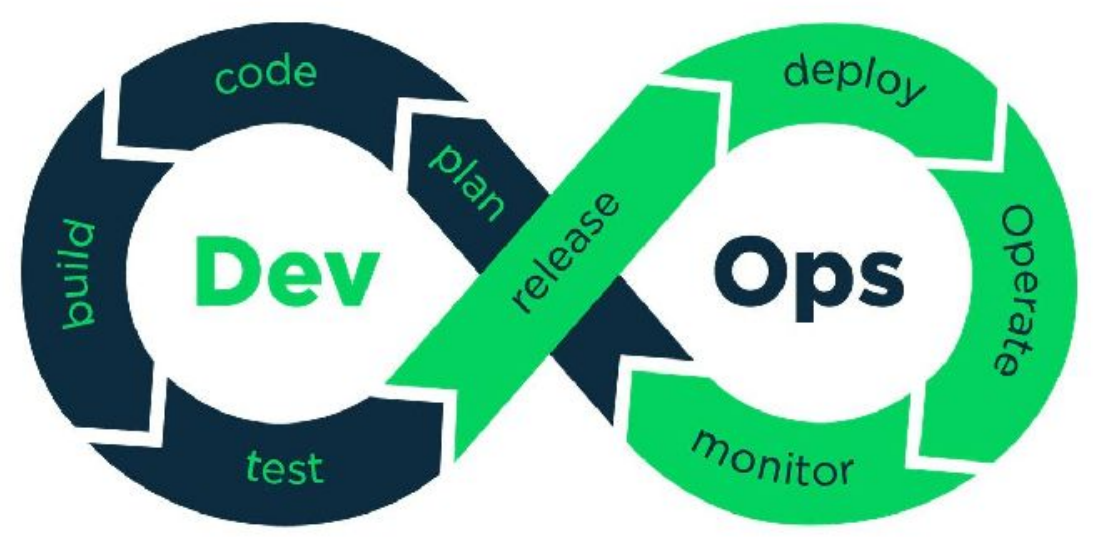

Fonte: Cole (2021)

O ciclo se inicia na fase de planejamento (PLAN) onde a equipe de desenvolvimento se reúne com os usuários demandantes com o objetivo de entender e iniciar o planejamento do que foi solicitado, seja um novo software ou a atualização de um existente. A próxima é a fase de codificação (CODE) quando os programadores vão preparar os códigos que comporão o programa. A fase de construção (BUILD) é a que ocorre a integração dos pedaços de códigos criados em etapas anteriores. A fase de teste (TEST) é considerada o ponto central, em que o programa que foi construído é testado a fim de encontrar algum tipo de erro no momento da execução. O programa passando da fase de teste vai para a de RELEASE, onde será disponibilizado para entrar em operação na fase de implantação (DEPLOY) só que em um ambiente controlado, após a equipe ter a certeza de que este está estável, o programa vai para a fase de operação (OPERATE) sendo colocada realmente em produção. Por último, mas não significando o fim, se inicia a fase de monitoramento (MONITOR) onde a equipe acompanha o desempenho da solução, recebe feedbacks dos usuários e se necessário reiniciar todo o ciclo caso receba uma solicitação de melhoria ou se a própria equipe perceba tal necessidade (YARLAGADDA, 2021).

Outro ponto importante a se destacar na cultura DevOps em comparação com a mentalidade existente atualmente nas empresas é com relação a mudanças de configuração ou de infraestrutura, a figura 2 destaca essa diferença (SHAH; DUBARIA, 2019). 
FIGURA 2: Diferença de mentalidade
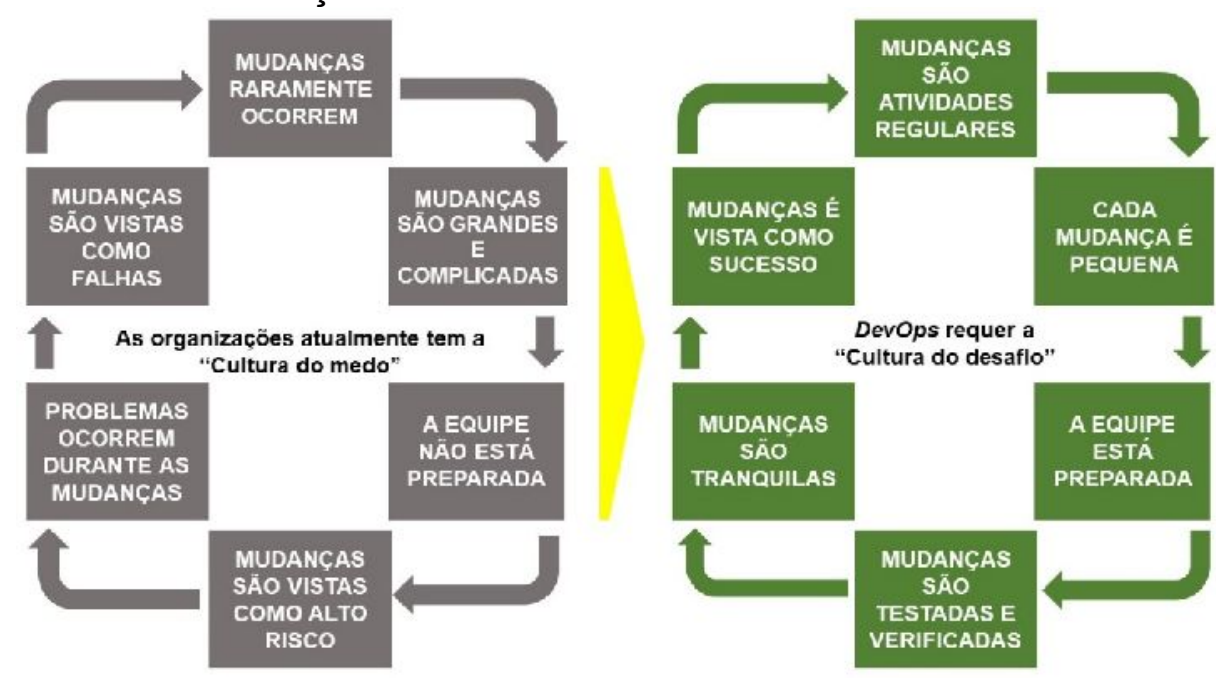

Fonte: Shah e Dubaria (2019)

Por fim, é importante destacar quatro aspectos que formam a prática do DevOps segundo Podgornik e Gibertoni (2020), o primeiro é a Cultura: priorizando pessoas a processos e ferramentas, pois softwares ou outras soluções são feitos por pessoas e para pessoas; segundo a Automatização: como característica essencial para que a prática DevOps gere ganhos expressivos para a corporação; em terceiro a Medição: por meio desta é possível aferir a qualidade; e por fim o Compartilhamento: DevOps cria uma cultura em que as pessoas compartilham ideias, processos e ferramentas.

\section{Infraestrutura como código}

Para início de discussão, o termo infraestrutura como um código do inglês Infrastructure as a Code - laaC ou simplesmente laC de Infrastructure as code, em uma definição simples, utiliza as ideias do $S D N$ para as redes clássicas, tentando resolver os problemas discutidos na subseção anterior, mas nesta seção esse conceito será mais detalhado.

A laaC faz com que as empresas possam gerenciar e manter todos os seus recursos de infraestrutura, como máquinas virtuais, firewalls e dispositivos de rede configuráveis via códigos padronizados em vez de processos e procedimentos de configuração manuais e individuais (JOHANN, 2017).

A ideia da laaC é tratar os recursos de infraestrutura como se fossem software em um código de programa, o que permite que a laaC utilize as práticas de desenvolvimento de software provisionando e implantando infraestrutura de rede rapidamente e consistente. A infraestrutura como código capacita a equipe de DevOps com ferramentas e recursos suficientes para implantar e manter a estrutura de rede demandada, podendo fazer mudanças rápidas na configuração de serviços e aplicativos, bem como automatizar tarefas repetitivas nesses recursos. Adicionalmente, a laaC pode ser integrada com o método de integração e entrega contínua, dentro de um pipeline, como se fosse uma esteira com vários procedimentos em sequência, que permite que o código a ser usado em um determinado equipamento possa ser automaticamente testado e implantado na 
infraestrutura sem interferência humana (JOHANN, 2017). Logo abaixo apresenta-se uma figura que explica esse pipeline.

FIGURA 3: Pipeline laaC

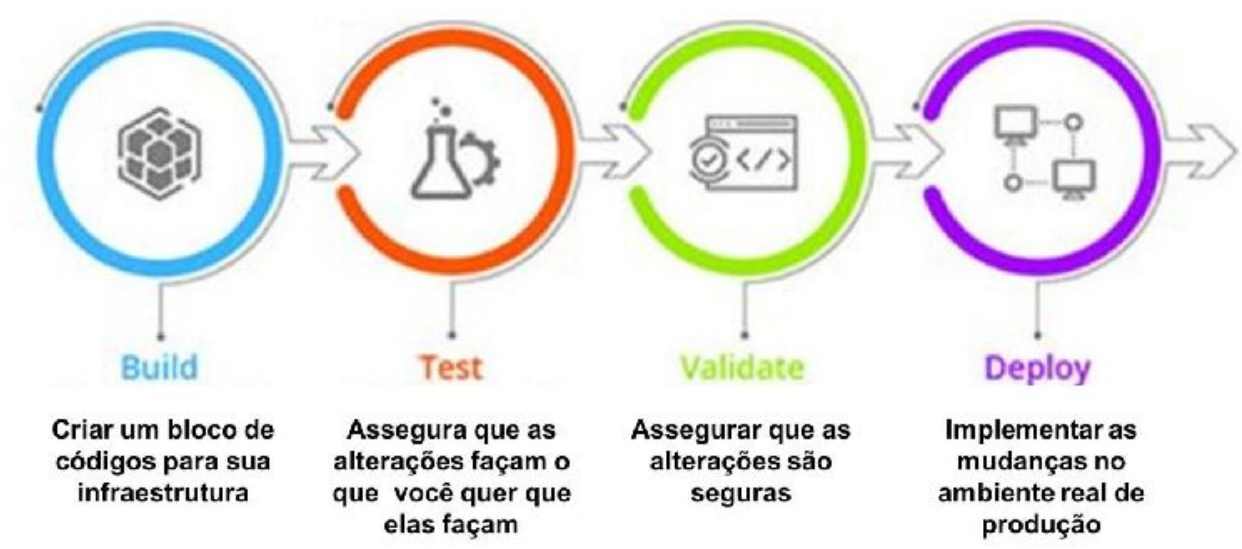

Fonte: Lewkowicz (2019)

A diferença entre SDN e laaC, segundo Johann (2017) em artigo publicado na revista IEEE Software, é que Redes definidas por software, SDN, formam um conjunto de ferramentas que permitem programar a infraestrutura localmente ou em plataformas nas nuvens como AWS [Amazon Web Services] ou Open-Stack, por exemplo. A infraestrutura como código expressa a filosofia de colocar as configurações dos seus equipamentos que formam a rede em arquivos de programas, ou seja, códigos, com controle de versão, executando testes automatizados para que essas configurações só sejam realmente disponibilizadas no ambiente de produção após a certeza que não produzirão nenhum tipo de falha.

\section{Controle de acesso}

O SDN tradicional e sobretudo o que é voltado para ambientes móveis trarão consigo muitas vantagens para o aprimoramento da $l o T$, mas além de tudo o que foi exposto como ganhos, trará grandes desafios na parte de segurança (CHICA et al., 2020). Uma das metodologias que podem ser aplicadas no ensino enfatiza tanto a fase de autenticação como a de autorização do usuário. Para a autenticação o padrão adotado no modelo a ser implementado será o $802.1 x$ como aplicado no trabalho de Matias et al., (2014), porém será comparado com outros padrões a fim de avaliar se essa é a melhor opção. Para a parte de autorização o padrão Rolebased Access Control - RBAC (AHN; SANDHU, 2000) será o modelo adotado inicialmente, mas com o decorrer de mais testes, poderá ser substituído por outro que forneça melhor resultado.

Com a necessidade crescente de acesso às informações com a mobilidade requisitada, os sistemas computacionais deverão proporcionar o acesso ao usuário levando em consideração vários aspectos como integridade, qualidade, veracidade dos dados, tempo de acesso, os direitos de acesso que o usuário possuirá dessa 
informação entre outros. A partir dessa problemática as redes sensíveis ao contexto surgem como solução adequada a esses requisitos (KANTER et al., 2014).

Para melhor definição, segue a seguinte definição de contexto:

Contexto é qualquer informação que pode ser usada para caracterizar a situação de uma entidade. Uma entidade pode ser uma pessoa, um lugar ou um objeto que seja considerado relevante para a interação entre um usuário e uma aplicação, incluindo-se o próprio usuário e a aplicação (PERERA et al., 2014 p. 420).

Segundo estes autores, o mecanismo que será construído baseado nesse referencial teórico apresentado, inicialmente se limitará a usar o contexto no sentido da presença ou não do usuário ativo no local onde é aplicado o controle de acesso, porém, quando este for estendido para loT, esse conceito deverá ser mais aprofundado com a criação de classe de contexto a fim de refinar as tomadas de decisão do sistema.

\section{Aprendizagem Baseada em Problemas - ABP}

A aprendizagem baseada em problemas (ABP) também muito referenciada pelo seu termo em inglês $P B L$ - Problem Based Learning é uma metodologia pedagógica que desafia os alunos a aprenderem via trabalhos cooperativos em grupos para desenvolver soluções de problemas do mundo real. O ponto central da ABP é o aluno, seu objetivo é fazer uso de problemas para que eles possam buscar soluções aplicando seus conhecimentos para encontrar resoluções. Assim, contribuindo para que eles desenvolvam habilidades de pensamento crítico e capacidade de resolver de forma ágil (CHEN et al., 2021).

FIGURA 4: Fluxo ABP

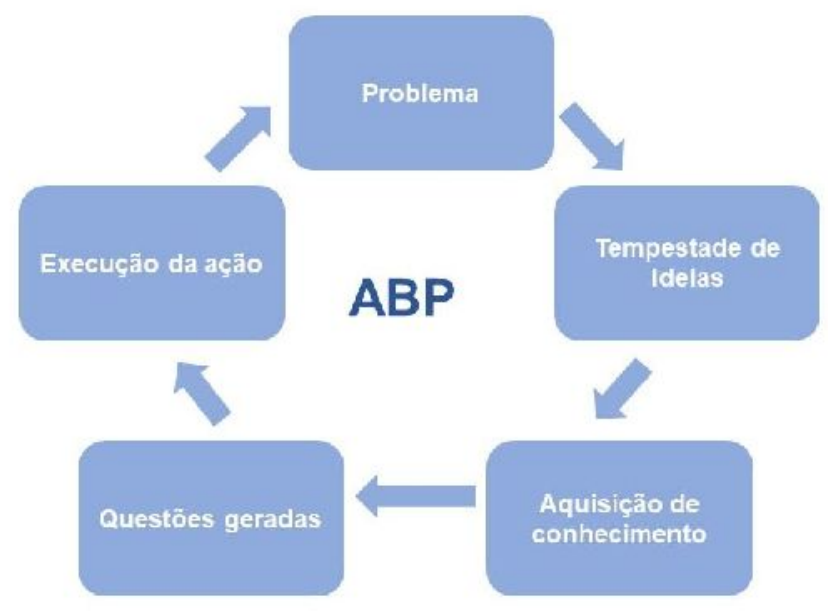

Fonte: Autoria própria

A prática da $\mathrm{ABP}$ gira em torno de um problema que é desenvolvido em paralelo com outra estratégia de ensino ativo conhecida como sala de aula invertida, onde os alunos irão adquirir conhecimentos por meio de pesquisas, seja em 
bibliotecas, internet ou em outros meios de informação, deixando de lado os paradigmas tradicionais do ensino presencial e fomentando a obtenção das soluções desejadas (CHEN et al., 2021). A figura acima resume a aplicação da ABP.

A ABP parte de um problema, que provoca a geração de ideias em busca pela solução por parte dos estudantes, que deverão adquirir conhecimento por intermédio de pesquisas, durante a aquisição do conhecimento questões serão geradas e nesse momento a colaboração entre os membros da equipe terá um papel importante pois, em conjunto, todos deverão direcionar as discussões para as melhores soluções, após a escolha da melhor, o grupo deve executar a ação utilizando o SCRUM a fim de entregar a solução do problema apresentado. (GONÇALVES et al., 2020).

Uma das práticas da ABP que é de suma importância como forma de expor à sociedade o que é feito na academia, é o entendimento de que o que é produzido pelos alunos deve transcender a utilidade meramente didática, é o fato de que os artefatos construídos em unidades de $\mathrm{ABP}$ devem ser submetidos à crítica externas e quem sabe serem aproveitados pelo mercado (FERREIRA; CANEDO, 2019).

Além de tudo exposto, é possível identificar no artigo de Subramaniam et al. (2017) que o SCRUM se destaca como o método muito aplicado em conjunto com a $A B P$, levando a acreditar que sua adoção vai ajudar na construção da autenticidade dos artefatos na ABP.

\section{SCRUM}

O termo ABP ou Aprendizado Baseado em Problemas muitas vezes é trocado por Aprendizagem Baseada em Projetos, pois no final o problema tem um princípio, um meio e um fim com a sua resolução, tendo todas as características de um projeto. Como destacada no final da subseção anterior, para reforçar a aprendizagem a metodologia escolhida ou conjunto de ferramentas foi o SCRUM.

Antes de iniciar a discussão sobre SCRUM se faz necessário falar antes sobre o manifesto ágil (MANIFESTO, 2021) que surgiu de um encontro de 17 profissionais que utilizavam métodos considerados ágeis em seus trabalhos para o desenvolvimento de software no ano de 2001, durante essa reunião ele puderam encontrar os pontos comuns que ajudavam suas formas de trabalho a serem bemsucedidas e concluíram que:

- Indivíduos e interações são mais importantes que processos e ferramentas;

- Software em funcionamento mais importantes que documentação abrangente;

- Colaboração com o cliente mais importante que negociação de contratos;

- Responder a mudanças é mais importante que seguir um plano.

Deve ser enfatizado que, mesmo o item à direita tendo menor valor do que o item a esquerda, isso não significa que ele será deixado de lado, este continua tendo importância. Desse manifesto sugiram 12 princípios que guiam esta forma de trabalhar:

1. A maior prioridade é satisfazer o cliente por meio da entrega cedo e frequente de software com valor. 
2. Mudanças de requisitos são bem-vindas, mesmo em fases tardias do desenvolvimento. Os processos Ágeis utilizam a mudança em favor da vantagem competitiva para o cliente.

3. Entregar software em funcionamento com frequência, desde a cada uma semana até a cada dois meses, com uma preferência por prazos mais curtos.

4. Os responsáveis pelo negócio e os desenvolvedores devem trabalhar em conjunto diariamente ao longo do projeto.

5. Construa projetos em torno de indivíduos motivados. Disponibilize o ambiente e o suporte que precisam e confie neles para realizarem o trabalho.

6. O método mais eficiente de transmitir informação para e entre uma equipe de desenvolvimento é a conversa face a face.

7. Software em funcionamento é a principal medida de progresso.

8. Os processos Ágeis promovem o desenvolvimento sustentável. Os patrocinadores, desenvolvedores e usuários devem ser capazes de manter indefinidamente um ritmo constante.

9. A atenção contínua à excelência técnica e a um bom projeto aumentam a agilidade.

10. A simplicidade (a arte de se maximizar a quantidade de trabalho realmente necessário para o projeto) é essencial.

11. As melhores arquiteturas, requisitos e projetos surgem de equipes que possuem a habilidade de se auto-organizarem.

12. Em intervalos de tempo regulares, a equipe reflete sobre como se tornar mais efetiva e então refina e ajusta seu comportamento de acordo.

Esses princípios que formam um conjunto de pensamentos amparados pelo Manifesto Ágil são o principal guia que norteia toda a nova cultura para empresa e instituições de ensino, vale salientar que apesar de focar no desenvolvimento de software, algumas ferramentas como o SCRUM podem ser aplicadas em outras áreas do conhecimento, como ocorreu em Bauerle et al. (2020).

Essa metodologia gira em torno de três pilares: A Transparência: Tudo é visível, disponível e conhecido por todos; a Inspeção: o trabalho é frequente e constantemente inspecionado; e a adaptação: para serem feitos ajustes rápidos e pequenas correções. Esses pilares formam a cultura que envolve o trabalho com o SCRUM e que se assemelha à cultura DevOps (CRUZ, 2013).

O SCRUM é um framework utilizado na gestão de projetos e desenvolvimento ágil de software e de outros tipos de projetos. Como mencionado anteriormente, os projetos devem ter um princípio, meio e fim, também deve estar focado em gerar valor através de entregas contínuas de software funcionando, possuindo suas entregas normalmente realizadas em ciclos de tempo fixo, do inglês time box, esses ciclos são chamados de sprint (CRUZ, 2013).

Antes de ser descrito os ritos que formam o SCRUM, deve ser entendido o papel das pessoas que irão trabalhar com essa ferramenta. Existem três funções que compõem a equipe SCRUM segundo Sutherland e Sutherland (2019):

1. Product Owner (P.O): É o responsável (apenas um por equipe) por manter um registro de todos os requisitos necessário para a entrega do produto a ser disponibilizado ao fim do projeto, conhecido como 
backlog do produto, detêm o conhecimento das regras de negócio, é responsável por priorizar as entregas e de que todos conheçam esses registros. É quem detém o conhecimento de negócio, quem faz a ponte com o mercado e com os clientes e que tem a visão de como o produto deve ficar ao final de todo o processo.

2. Scrum Master: É o responsável (também um por equipe) por garantir que todos estejam aderentes aos processos inerentes ao SCRUM, sigam as suas regras, participem das cerimônias e usem os artefatos corretamente. É quem ensina e corrige tal qual como prescrito no SCRUM. É quem puxa a melhoria contínua e remove qualquer tipo de obstáculo que possa atrapalhar a execução do trabalho pela equipe de desenvolvimento.

3. Time de desenvolvimento: são todos os outros membros da equipe SCRUM. Entende-se que todos que contribuem para 0 desenvolvimento do produto sejam chamados de desenvolvedores, ou seja, não necessariamente são programadores, não importando os seus cargos ou conhecimentos individuais. É importante que o time tenha tantos componentes quanto necessário para fazer a entrega acontecer, mas não muitos a ponto de prejudicar o processo, a maioria dos profissionais que usam essa metodologia sugerem entre três e nove participantes por time. Essa equipe precisa ser multidisciplinar, possuir conhecimentos variados, ser auto-organizável, pois no SCRUM não existe a figura central de um chefe e é o time que vai definir o tempo da sprint e o que será trabalhado dentro dela. Todo o ritual do SCRUM é demonstrado na figura 5.

FIGURA 5: Rito SCRUM

\section{FRAMEWORK SCRUM}

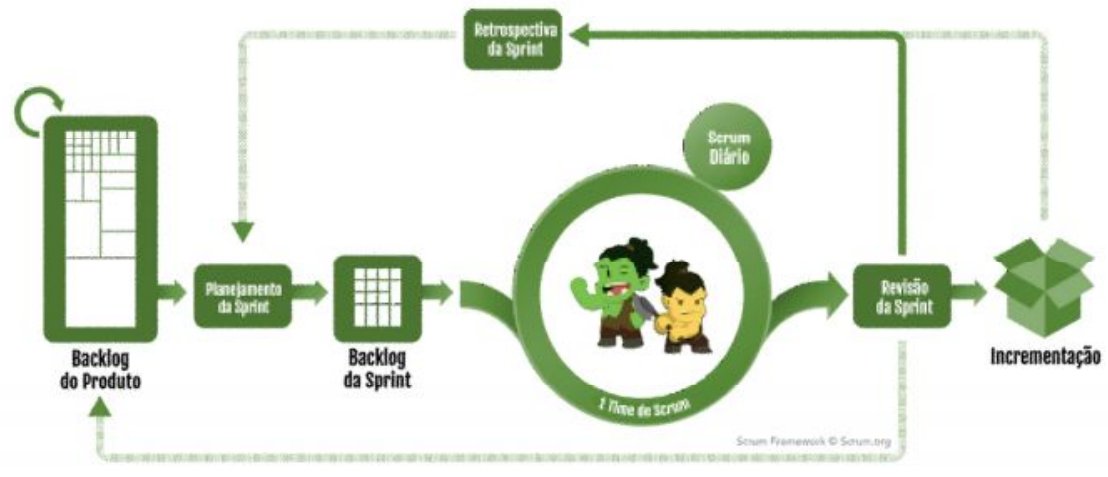

Fonte: Orcestra (2020)

O backlog do produto possui todos os requisitos, tarefas e atividades necessárias para que o projeto seja entregue, sendo responsabilidade do P.O nortear o time para todos os componentes existentes nessa lista ao final correspondam ao projeto demandado pelo cliente. A parte de priorização de cada atividade também é descrita no backlog ficando a cargo do P.O em conjunto com o time a definição da ordem de prioridade dos elementos a disposição (CRUZ, 2013). 
No Planejamento da sprint o P.O apresenta o backlog do produto, explicando ao time quais são os objetivos do projeto e o que eles deveriam entregar primeiro para gerar valor ao cliente o mais rápido possível. Baseado nessas explicações, o Time de Desenvolvimento define os itens mais prioritários que estarão especificados a nível de negócio e técnico de forma que permita com que eles consigam gerar estimativas de esforço e tempo que facilitarão o trabalho. Quando as estimativas preencherem todo o tempo que terão no desenvolvimento na sprint, o time fecha o escopo, apresenta ao P.O o backlog da sprint e seu conjunto de atividade, e não havendo objeções, o desenvolvimento começa. O Scrum Master é o responsável por garantir e geralmente conduzir esta cerimônia (CRUZ, 2013).

O círculo central representa a sprint que é o tempo definido pelo Time de desenvolvimento para executar parte do projeto, vale lembrar que ela tem um tempo fixo de execução e não é permitido que ele seja modificado. Dentro da sprint tem-se a daily Meeting ou scrum diário, uma curta reunião diária que deve ocorrer a fim de deixar todos os membros cientes do que cada um fez no dia anterior, o que vão fazer no dia atual e quais foram as dificuldades encontradas no intuito de que o Scrum Master possa atuar para resolver tais empecilhos (CRUZ, 2013).

Ao término de cada sprint, no último dia, ocorre uma reunião conhecida como Revisão da sprint. Essa é uma cerimônia de prestação de contas, onde o time apresenta os avanços da sprint para o P.O e demais apoiadores do projeto. Nessa apresentação o time receberá feedbacks que permitirão corrigir qualquer desvio de curso e que poderão também gerar melhorias no projeto não previstas inicialmente no planejamento inicial, o que torna o SCRUM um excelente método para produtos inovadores e complexos, pois o time ajusta-se constantemente às mudanças (CRUZ, 2013).

Após a Revisão da sprint, é hora da Retrospectiva da sprint, a cerimônia de adaptação do processo de desenvolvimento do time, promovendo a melhoria contínua. Aqui o time irá discutir pontos bons e ruins que ocorreram na última sprint no que tange pessoas, práticas, ferramentas e tudo mais que possa surgir no encontro e que tenha margem para melhoria. Diversas são as dinâmicas que o Scrum Master pode usar para conduzir esta cerimônia, mas todas elas levarão a melhorias no processo ao final (CRUZ, 2013).

\section{TRABALHOS RELACIONADOS}

Muitas universidades estão trabalhando, para adequar seus cursos ao paradigma $S D N$, inserindo novas disciplinas ou cursos de extensão. Desse modo, será apresentada uma breve revisão da literatura que discorre a respeito de algumas experiências nessa linha que possibilitam discutir o ensino de $S D N$ alinhado à metodologia de ensino Aprendizagem baseada em problemas e ao SCRUM.

No artigo apresentado por Suh et al. (2017) os autores propõem a implementação de um curso onde os alunos são encorajados a projetar e gerenciar redes baseadas em $S D N$, no lugar de focar no aprendizado de fabricantes de equipamentos de rede específicos. Eles afirmam que devido o $S D N$ ser novo e ainda estar em desenvolvimento, o curso corre o risco de rapidamente ficar obsoleto, justificando assim o foco na parte conceitual para que os alunos possam estar preparados para novas atualizações na tecnologia que possam surgir. A forma de ensino apresentada uniu a parte teórica com atividades práticas através da 
aprendizagem baseado em problemas - ABP. Os autores ressaltam que o curso precisa de uma composição híbrida entre ambientes virtuais e equipamentos reais.

Em outro artigo relacionado com SDN, Caicedo et al. (2019) referenciam o trabalhado discutido no parágrafo anterior, mas não só implementam um curso, eles inserem-no dentro da universidade em forma de uma metodologia que abrange os cursos de graduação, mestrado e doutorado. Essa aplicação faz com que o conhecimento transborde para o mercado por intermédio das pesquisas aplicadas. Sua metodologia de ensino faz uso de laboratórios práticos e virtuais, assim como da ABP.

Com relação ao DevOps, alguns artigos utilizam a maneira de trabalhar dentro dessa cultura para preparar os alunos tanto para o desenvolvimento de novos programas como para a operação de equipamentos em nuvem. No trabalho de Bobrov et al. (2020) os autores focam no desenvolvimento de software utilizando ferramentas e orientações advindas do DevOps em conjunto com metodologias de desenvolvimento ágil, com enfoque na ferramenta SCRUM.

No trabalho apresentado por Ghantous e Gill (2019) os autores enfatizam a importância do uso de ferramentas ágeis em conjunto com o DevOps para uma melhor preparação dos estudantes, eles ainda aplicam uma metodologia de ensino chamada de teoria do aprendizado colaborativo de 1993 que possui muitas similaridades com a ABP.

Como base na revisão bibliográfica, é apresentada uma proposta baseada nas principais vantagens encontradas nos artigos da revisão, considerando também economia financeira de recursos no trabalho proposto, pois se utilizam práticas em laboratórios virtuais, SDN em conjunto com equipamentos clássicos (não compatíveis com $S D N$, por intermédio da infraestrutura como código - laaC, permitindo que o aprendizado possa ser condizente com os requisitos demandados pelo mercado de tecnologia atual e futuro.

\section{CONSIDERAÇÕES FINAIS}

A demanda por programadores e por infraestrutura em nuvem cresce muito e não é de agora, e com a situação de pandemia vivenciada no mundo, o acesso rápido e confiável à Internet é imprescindível, assim a aplicação de uma metodologia que possa capacitar os alunos a serem programadores, operadores de infraestrutura tanto de rede como de computadores e trabalhando em equipe de forma ágil de forma remota é algo muito valorizado no mercado de trabalho atual, porém muitos cursos ainda preparam profissionais ou em desenvolvimento ou em operação, mantendo a falta de eficiência requisitada atualmente pelas grandes empresas. Assim, ao fim da revisão da literatura, observou-se que o ensino de SDN alinhado à metodologia de ensino Aprendizagem baseada em problemas e ao SCRUM contribui para o desenvolvimento de competências e habilidades relacionadas à pesquisa e inovação de produtos educacionais e tecnológicos. Portanto, as propostas didáticas visam estimular nos atores envolvidos no processo de ensino e aprendizagem:

- Pensamento crítico para resolução de problemas, tendo a habilidade de compartilhar pensamentos, questões, ideias e soluções sobre um determinado aspecto;

- Comunicação efetiva com a habilidade de realizar trabalhos em conjunto para alcançar um objetivo, permitindo a união do talento individual, experiência de vida e inteligência na proposta de soluções; 
- Colaboração para permitir olhar um problema de uma forma diferente e assim, unir diversas áreas e assuntos para resolvê-lo;

- Criatividade e Inovação fornecendo a habilidade de tentar criar abordagens diferentes em busca de soluções.

\section{REFERÊNCIAS}

AHN, G.-J.; SANDHU, R.. Role-based authorization constraints specification. ACM Transactions on Information and System Security (TISSEC), ACM, New York, NY, USA, v. 3, n. 4, p. 207-226, 2000. Disponível em: <https://doi.org/10.1145/382912.382913>. Acesso em: 10 ago. 2021.

BASHER, M.. DevOps: An explorative case study on the challenges and opportunities in implementing Infrastructure as code. 2019. Disponível em: $<$ https://www.diva-portal.org/smash/record.jsf?pid=diva2\%3A1331526\&dswid=4115>. Acesso em: 10 ago. 2021.

BAUERLE, B. V.; ZIMMERMANN, F.; QUINTANS. F. C. F.; DOSTLER, P. M. G.. Gestão da Inovação na Rede Federal de Educação Profissional, Científica e Tecnológica. Bahia: Editora do Instituto Federal de Educação, Ciência e Tecnologia da $\quad$ Bahia, $2020 . \quad 41 \quad$ p. $\quad$ Disponível em $<$ https://portal.ifba.edu.br/prpgi/editora/livros/multidisciplinar/gestao-de-inovacao-narede-federal>. Acesso em: 10 ago. 2021.

BENZEKKI, K.; FERGOUGUI, A. E.; ELALAOUI, A. E.. Software-defined networking (sdn): a survey. Security and Communication Networks, v. 9, n. 18, p. 5803-5833, 2016. Disponível em: <https://onlinelibrary.wiley.com/doi/abs/10.1002/sec.1737>. Acesso em: 10 ago. 2021.

BRITO, I., GRAMACHO, S., FERREIRA, I., NAZARÉ, M., SAMPAIO, L., FIGUEIREDO, G. B.. OpenWiMesh: A framework for software defined wireless mesh networks. In: 2014 Brazilian Symposium on Computer Networks and Distributed Systems. IEEE, 2014. p. 199-206. Disponível em: <https://doi.org/10.1109/SBRC.2014.24>. Acesso em: 17 set. 2021.

BOBROV, E.; BUCCHIARONE, A.; CAPOZUCCA, A.; GUELFI, N.; MAZZARA, M. et al.. Devops and its philosophy: Education matters! In: Microservices. [S.I.]: Springer, 2020. p. 349-361. Disponível em: < https://doi.org/10.1007/978-3-030-31646-4_14 >. Acesso em: 11 ago. 2021.

CAICEDO, C. V.; PRIETO, Y.; PEZOA, J. E.; SOBARZO, S. K.; GHANI, N.. A novel framework for sdn teaching and research: A chilean university case study. IEEE Communications Magazine, IEEE, v. 57, n. 11, p. 67-73, 2019. Disponível em: <https://doi.org/10.1109/MCOM.001.1900261>. Acesso em: 10 ago. 2021.

CARAGUAY, A. L. V.; PERAL, A. B.; LÓPEZ, L. I. B.; VILLALBA, L. J. G.. Sdn: Evolution and opportunities in the development iot applications. International Journal of Distributed Sensor Networks, Hindawi Publishing Corporation, v. 2014, 
2014. Disponível em: <https://doi.org/10.1155\%2F2014\%2F735142 >. Acesso em: 11 de ago. 2021.

CHEN, J.; KOLMOS, A.; DU, X.. Forms of implementation and challenges of pbl in engineering education: a review of literature. European Journal of Engineering Education, Taylor \& Francis, v. 46, n. 1, p. 90-115, 2021. Disponível em: $<$ https://doi.org/10.1080/03043797.2020.1718615 >. Acesso em: 11 ago. 2021.

CHICA, J. C. C.; IMBACHI, J. C.; VEGA, J. F. B.. Security in sdn: A comprehensive survey. Journal of Network and Computer Applications, Elsevier, v. 159, p. 102595, 2020. Disponível em: <http://hdl.handle.net/11407/5743>. Acesso em: 10 ago. 2021.

COLE, A.. Como o DevOps afeta o trabalho do CIO. Mandic, 2021. Disponível em: < https://blog.mandic.com.br/artigos/como-o-devops-afeta-o-trabalho-do-cio/>. Acesso em: 10 ago. 2021.

CRUZ, F.. Scrum e Pmbok Unidos no Gerenciamento de Projetos. 1.ed. São Paulo: Brasport, 2013.

DYCK, A.; PENNERS, R.; LICHTER, H.. Towards definitions for release engineering and DevOps. In: 2015 IEEE/ACM 3rd International Workshop on Release Engineering. IEEE, p. 3-3,2015. Disponível em: <https://doi.org/10.1109/RELENG.2015.10>. Acesso em: 17 set. 2021.

FERREIRA, V.; CANEDO, E.. Autenticidade na aprendizagem baseada em projetos para desenvolvimento de software: Uma Revisão Sistemática de Literatura. In: Brazilian Symposium on Computers in Education (Simpósio Brasileiro de Informática na Educação-SBIE). 2019.2 Disponível em: <http://dx.doi.org/10.5753/cbie.sbie.2019.41>. Acesso em: 17 set. 2021.

GHANTOUS, G. B.; GILL, A. Q.. An agile-devops reference architecture for teaching enterprise agile. International Journal of Learning, Teaching and Educational Research, 2019. Disponível em: <http://hdl.handle.net/10453/135151>. Acesso em: 11 ago. 2021.

GIL, A. C.. Como elaborar projetos de pesquisa. 5. ed. São Paulo: Atlas, 2008.

GONÇALVES, M. F.; GONÇALVES, A. M.; GONÇALVES, I. M. F.. Aprendizagem baseada em problemas: uma abordagem no ensino superior na área da saúde. Práticas Educativas, Memórias e Oralidades-Rev. Pemo, v. 2, n. 1, p. 1-12, 2020. Disponível em: < https://doi.org/10.47149/pemo.v2i1.3676 >. Acesso em: 17 set. 2021.

GUDE, N.; PFAFF, B.; KOPONEN, T.; CASADO, M.; SHENKER, S. et al.. Nox: towards an operating system for networks. ACM SIGCOMM Computer Communication Review, ACM, v. 38, n. 3, p. 105-110, 2008. Disponível em: https://dl.acm.org/doi/pdf/10.1145/1384609.1384625?casa_token=drWUDf0ck70AAA 
AA:z-i4AFg60Z8RiRtPhvgvTPcHOcWpus3MDukLTng4L9VJ4Fcc9V3RNtBI9WANiUqQprA4F1SK6zY_A. Acesso em: 23 ago. 2021.

JOHANN, S.. Kief morris on infrastructure as code. IEEE Software, IEEE Computer Society. 34, n. 01, p. 117-120, 2017. Disponível em: <https://doi.ieeecomputersociety.org/10.1109/MS.2017.13>. Acesso em: 10 ago. 2021.

KANTER, T.; RAHMANI, R.; MAHMUD, A.. Conceptual framework for internet of things' virtualization via openflow in context-aware networks. arXiv preprint arXiv:1401.7437, 2014. Disponível em: <https://arxiv.org/abs/1401.7437v1>. Acesso em: 10 ago. 2021.

LEWKOWICZ, J.. Itential launches new API services for Network Infrastructure as Code. It Ops times, 2019. Disponível em: $<$ https://www.itopstimes.com/devops/itential-launches-new-api-services-for-networkinfrastructure-as-code/>. Acesso em: 10 ago. 2021.

LEITE, L., ROCHA, C., KON, F., MILOJICIC, D., MEIRELLES, P.. A survey of DevOps concepts and challenges. ACM Computing Surveys (CSUR), v. 52, n. 6, p. 1-35, 2019. Disponível em: <https://doi.org/10.1145/3359981>. Acesso em: 17 set. 2021.

MANIFESTO. Manifesto for Agile Software Development, 2021. Disponível em: $<$ https://agilemanifesto.org/>. Acesso em: 10 ago. 2021.

MATIAS, J.; GARAY, J.; MENDIOLA, A; TOLEDO, N; JACOB, E.. Flownac: Flowbased network access control. In: IEEE. Software Defined Networks (EWSDN), 2014 Third European Workshop on. [S.I.], 2014. Disponível em: <https://doi.org/10.1109/EWSDN.2014.39>. Acesso em: 23 ago. 2021.

MCKEOWN, N.; ANDERSON, T. E.; BALAKRISHNAN, H.; PARULKAR, G.; PETERSON, L.L. et al.. Openflow: enabling innovation in campus networks. ACM SIGCOMM Computer Communication Review, ACM, v. 38, n. 2, p. 69-74, 2008. Disponível em: <https://doi.org/10.1145/1355734.1355746>. Acesso em: 10 ago. 2021.

OPENDAYLIGHT. OPENDAYLIGHT, 2021. Disponível em: <http://www.opendaylight.org>. Acesso em: 10 ago. 2021.

ORCESTRA. Scrum: Como fazer mais em menos tempo. Disponível em: <https://www.orcestra.com.br/post/scrum-como-fazer-mais-em-menos-tempo>.

Acesso em: 10 ago. 2021.

PERERA, C.; ZASLAVSKY, A.; CHRISTEN, P.; GEORGAKOPOULOS, D.. Context aware computing for the internet of things: A survey. Communications Surveys \& Tutorials, IEEE, IEEE, v. 16, n. 1, p. 414-454, 2014. Disponível em: <https://doi.org/10.1109/SURV.2013.042313.00197>. Acesso em: 10 ago. 2021. 
PODGORNIK, H.; GIBERTONI, D.. Um estudo de caso sobre o desenvolvimento de uma aplicação scrum e devops. Revista Interface Tecnológica, v. 17, n. 2, p. 244255, 2020. Disponível em: <https://doi.org/10.31510/infa.v17i2.978>. Acesso em: 10 ago. 2021.

POX. noxrepo (NOX Repo), 2021. Disponível em: <https://github.com/noxrepo/>. Acesso em: 10 ago. 2021.

RAY, P. P.; KUMAR, N.. Sdn/nfv architectures for edge-cloud oriented iot: A systematic review. Computer Communications, v. 169, p. 129-153, 2021. ISSN 0140-3664. Disponível em: <https://www.sciencedirect.com/science/article/pii/S0140366421000396>. Acesso em: 23 ago. 2021.

SHAH, J. A.; DUBARIA, D.. Netdevops: A new era towards networking \& devops. In: 2019 IEEE 10th Annual Ubiquitous Computing, Electronics \& Mobile Communication Conference (UEMCON). [S.I.], 2019. p. 0775-0779. Disponível em: < https://doi.org/10.1109/UEMCON47517.2019.8992969>. Acesso em: 10 ago. 2021.

SUBRAMANIAM, S.; CHUA, F.-F.; CHAN, G.-Y.. Project-based learning for software engineering - an implementation framework. Journal of Telecommunication, Electronic and Computer Engineering (JTEC), v. 9, n. 3-4, p. 81-85, 2017. Disponível em: <https://jtec.utem.edu.my/jtec/article/view/2922>. Acesso em: 12 aug. 2021.

SUH, J.; BOJOVIĆ, Ž.; DESPOTOVIĆ-ZRAKIĆ, M.; BOGDANOVIĆ, Z.; LABUS, A.. Designing a course and infrastructure for teaching software-defined networking. Computer Applications in Engineering Education, Wiley Online Library, v. 25, n. 4, p. 554-567,2017. Disponível em: <https://doi.org/10.1002/cae.21820>. Acesso em: 10 ago. 2021.

SUTHERLAND, J.; SUTHERLAND, J. J.. SCRUM: a arte de fazer o dobro do trabalho na metade do tempo. 1.ed. Rio de Janeiro: Sextante, 2019.

YARLAGADDA, R. T.. DevOps and Its Practices. International Journal of Creative Research Thoughts (IJCRT), ISSN, p. 2320-2882, 2021. Disponível em: <http://www.ijcrt.org/papers/lJCRT2103016.pdf>. Acesso em: 17 set. 2021. 